\title{
Elastic Pekeris waveguide normal mode solution comparisons against laboratory data
}

Joseph D. Schneiderwind, Jon M. Collis, and Harry J. Simpson

Citation: The Journal of the Acoustical Society of America 132, EL182 (2012); doi: 10.1121/1.4740227

View online: https://doi.org/10.1121/1.4740227

View Table of Contents: https://asa.scitation.org/toc/jas/132/3

Published by the Acoustical Society of America

\section{ARTICLES YOU MAY BE INTERESTED IN}

On the acoustic field in a Pekeris waveguide with attenuation in the bottom half-space

The Journal of the Acoustical Society of America 119, 123 (2006); https://doi.org/10.1121/1.2141212

The Pekeris waveguide revisited

The Journal of the Acoustical Society of America 120, 1183 (2006); https://doi.org/10.1121/1.2221536

A normal mode model for acousto-elastic ocean environments

The Journal of the Acoustical Society of America 100, 3631 (1996); https://doi.org/10.1121/1.417226

Estimating the sound speed of a shallow-water marine sediment from the head wave excited by a low-flying helicopter

The Journal of the Acoustical Society of America 142, 2273 (2017); https://doi.org/10.1121/1.5007953

The effective depth of a Pekeris ocean waveguide, including shear wave effects

The Journal of the Acoustical Society of America 85, 648 (1989); https://doi.org/10.1121/1.397590

A coupled mode solution for acoustic propagation in a waveguide with stepwise depth variations of a penetrable bottom

The Journal of the Acoustical Society of America 74, 188 (1983); https://doi.org/10.1121/1.389707 


\title{
Elastic Pekeris waveguide normal mode solution comparisons against laboratory data
}

\author{
Joseph D. Schneiderwind ${ }^{\text {a) }}$ \\ CINTAL, Centro de Investigação Tecnológica do Algarve, \\ University do Algarve, Campus de Gambelas 8005-139 Faro, Portugal \\ jdschneiderwind@gmail.com \\ Jon M. Collis \\ Department of Applied Mathematics and Statistics, \\ Colorado School of Mines, Golden, Colorado 80401 \\ jcollis@mines.edu \\ Harry J. Simpson \\ Physical Acoustic Branch Code 7136, Naval Research Laboratory, \\ 4555 Overlook Avenue, SW, Washington DC 20375 \\ harry.simpson@nrl.navy.mil
}

\begin{abstract}
Following the derivation presented by Press and Ewing [Geophysics 15, 426-446 (1950)], a normal mode solution for the Pekeris waveguide problem with an elastic bottom is outlined. The analytic solution is benchmarked against data collected in an experiment performed at the Naval Research Laboratory [Collis et al., J. Acoust. Soc. Am. 122, 1987-1993 (2007)]. Comparisons reveal a close match between the analytic solution and experimental data. Results are strongly dependent on the accuracy of the horizontal wavenumbers for the modes, and horizontal wavenumber spectra are compared against those from the experimental data.

(C) 2012 Acoustical Society of America

PACS numbers: 43.30.Bp, 43.30.Ma, 43.30.Zk [WS]

Date Received: February 16, 2012 Date Accepted: July 17, 2012
\end{abstract}

\section{Introduction}

Ocean acoustic studies generally assume that the ocean bottom is fluid, and normal mode solutions for seismo-acoustic environments have received limited attention because effects due to elasticity in the bottom may not be a significant factor in many propagation scenarios. ${ }^{1}$ The seismo-acoustic environment considered here, that of an oceanic fluid layer overlying an elastic half space, will be referred to as the elastic Pekeris waveguide. In this paper, we compare normal mode solutions for the elastic Pekeris waveguide problem against data from a seismo-acoustic tank experiment performed in 2004 at the Naval Research Laboratory. ${ }^{2}$ Close agreement between normal mode solution predictions and measured experimental data is obtained for nearly range-independent propagation problems.

\section{Normal mode solution}

Among the first mathematical solutions to a shallow-water acoustic propagation problem was the Pekeris waveguide in $1948 .^{3}$ This waveguide was extended to include an elastic solid bottom by Press and Ewing in $1950,{ }^{4}$ and it is their solution that is employed here. The bottom is assumed to be an isospeed solid half space of constant density $\rho_{2}$, compressional wave speed $c_{2}$, and shear wave speed $c_{s}$. The water layer assumes constant compressional wave speed $c_{1}$ and density $\rho_{1}$. Field and environmental quantities will be

\footnotetext{
${ }^{\text {a) }}$ Author to whom correspondence should be addressed.
} 
denoted by a subscript (1) in the water, (2) in the bottom, and quantities associated with the shear wave field by an (s). An axially symmetric cylindrical coordinate geometry is assumed, the $z$-coordinate positive downwards, with the waveguide boundaries assumed planar and parallel. A time-harmonic point source of angular frequency $\omega$ and time dependence $\exp (-i \omega t)$ is assumed in the water at depth $z_{s}<H$, with $H$ the depth of the water.

Pressure fields are determined from the compressional and shear displacement potentials, $\phi(r, z)$ and $\psi(r, z)$, by

$$
\begin{gathered}
p_{1}=\rho_{1} \omega^{2} \phi_{1}, \quad p_{2}=\frac{\rho_{2} c_{2}^{2}}{2} \Delta_{2}, \\
\Delta_{2}=\frac{1}{r} \frac{\partial}{\partial r}\left(r u_{2}\right)+\frac{\partial w_{2}}{\partial z}, \quad u_{2}=\frac{\partial \phi_{2}}{\partial r}+\frac{\partial^{2} \psi}{\partial r \partial z}, \quad w_{2}=\frac{\partial \phi_{2}}{\partial z}+\frac{\partial^{2} \psi}{\partial z^{2}}+k_{s}^{2} \psi,
\end{gathered}
$$

where $\Delta$ is the dilatation, $p$ is the acoustic pressure, $u$ is the horizontal particle displacement in the $r$ direction, $w$ is the vertical particle displacement, and $k$ is the medium wavenumber. The potentials are solutions to the Helmholtz equations

$$
\begin{gathered}
\left(\nabla^{2}+k_{j}^{2}\right) \phi_{j}=0, \quad j=1,2, \\
\left(\nabla^{2}+k_{s}^{2}\right) \psi=0,
\end{gathered}
$$

for medium wavenumbers $k_{j}=\omega / c_{j}$ and $k_{s}=\omega / c_{s}$, with the $\nabla^{2}$ operator in cylindrical coordinates. Loss in the bottom is included by using complex wave speeds $C_{2}=c_{2} /\left(1+i \eta \alpha_{p}\right)$ and $C_{s}=c_{s} /\left(1+i \eta \alpha_{s}\right),{ }^{5}$ where $\alpha_{p}$ and $\alpha_{s}$ are the compressional and shear wave attenuations in decibels per wavelength, and $\eta=\left(40 \pi \log _{10} e\right)^{-1}$. The potentials must satisfy a pressure-release surface boundary condition, and continuity of vertical particle displacement, normal stress, and tangential stress at the bottom interface

$$
\begin{gathered}
\phi_{1}=0, \quad \text { at } z=0, \\
w_{1}=w_{2}, \quad\left(\sigma_{z z}\right)_{1}=\left(\sigma_{z z}\right)_{2}, \quad\left(\sigma_{z r}\right)_{2}=0, \quad \text { at } z=H,
\end{gathered}
$$

with the normal and tangential stresses given by

$$
\sigma_{z z}=\lambda \nabla^{2} \phi+2 \mu \frac{\partial w}{\partial z}, \quad \sigma_{z r}=\mu\left(\frac{\partial u}{\partial z}+\frac{\partial w}{\partial r}\right)
$$

where $\lambda$ and $\mu$ are Lamé constants.

Boundary conditions are satisfied explicitly, and the point source singularity is treated using the approach of Ref. 4, which involves separating the fluid layer into two layers at the source depth and applying continuity of pressure and discontinuity of vertical displacement across this interface. The particle displacement potential above the source is denoted without a prime as $\phi_{1}$, and the potential below the source is denoted with a prime as $\phi_{1}^{\prime}$. The particle displacement potentials satisfying the two-dimensional Helmholtz equations and boundary conditions are of the form

$$
\begin{gathered}
\phi_{1}=2 \int_{0}^{\infty}\left[A \sin \left(k_{z, 1} z\right) J_{0}\left(k_{r} r\right)\right] k_{r} d k_{r}, \quad 0 \leq z \leq z_{s}, \\
\phi_{1}^{\prime}=2 \int_{0}^{\infty}\left[\left(B \sin \left(k_{z, 1} z\right)+C \cos \left(k_{z, 1} z\right)\right) J_{0}\left(k_{r} r\right)\right] k_{r} d k_{r}, \quad z_{s} \leq z \leq H, \\
\phi_{2}=2 \int_{0}^{\infty}\left[D e^{-k_{z}, 2^{z}} J_{0}\left(k_{r} r\right)\right] k_{r} d k_{r}, \quad z>H,
\end{gathered}
$$




$$
\psi=2 \int_{0}^{\infty}\left[E e^{-k_{z}, s^{z}} J_{0}\left(k_{r} r\right)\right] k_{r} d k_{r}, \quad z>H
$$

where $k_{r}, k_{z, 1}, k_{z, 2}$, and $k_{z, s}$ are found by substituting the arguments of Eqs. (7)-(10) into the respective Helmholtz equations, and $A, B, C, D$, and $E$ are constants determined by the boundary and interface conditions. The vertical and horizontal wavenumber components in the fluid are related to the medium wavenumber by $k_{z, 1}=\left(k_{1}^{2}-k_{r}^{2}\right)^{1 / 2}$. In the bottom, $k_{z, 2}$ and $k_{z, s}$ are defined following the convention ${ }^{3}$ of Pekeris

$$
k_{z, 2}=\left\{\begin{array}{ll}
-i \sqrt{k_{2}^{2}-k_{r}^{2}}, & \left|k_{r}\right|<k_{2} \\
\sqrt{k_{r}^{2}-k_{2}^{2}}, & \left|k_{r}\right|>k_{2}
\end{array} \text { and } k_{z, s}= \begin{cases}-i \sqrt{k_{s}^{2}-k_{r}^{2}}, & \left|k_{r}\right|<k_{2} \\
\sqrt{k_{r}^{2}-k_{s}^{2}}, & \left|k_{r}\right|>k_{2},\end{cases}\right.
$$

to satisfy the radiation condition for $z \rightarrow \infty$.

Applying boundary and interface conditions results in a linear system with a solution having poles for values of the horizontal wavenumber where the determinant of the coefficient matrix vanishes. The determinant for the elastic Pekeris waveguide is given by

$$
\operatorname{det}\left(k_{r}\right)=\frac{\rho_{1}}{\rho_{2}} \frac{\omega^{4}}{c_{s}^{4}} \frac{k_{z, 2}}{k_{z, 1}} \tan \left(k_{z, 1} H\right)-\left[4 k_{r}^{2} k_{z, 2} k_{z, s}-\left(2 k_{r}^{2}-\omega^{2} / c_{s}^{2}\right)^{2}\right] .
$$

The transcendental equation $\operatorname{det}\left(k_{r}\right)=0$ is referred to as the characteristic equation for this problem and its roots are found numerically. For the elastic Pekeris waveguide problem, finding these complex roots accurately is challenging. A useful discussion and analysis of root finding for the elastic Pekeris waveguide problem can be found in Ref. 6 .

The integrals in Eqs. (7)-(10) can be expressed exactly as the sum of a contour integral and two branch line integrals corresponding to the branch points $k_{r}=k_{1}$ and $k_{r}=k_{2} .{ }^{3,7}$ The contour integral is evaluated as a sum of residues, ${ }^{8}$ which vary as $r^{-1 / 2}$ and give the normal mode solution, while the branch line integrals (continuum contribution) vary as $r^{-2}$ and are neglected by assuming $k r \gg 1$ (far field assumption). Finding roots to the characteristic equation locates the residues and yields the horizontal wavenumbers for the system $k_{r n}$.

Writing the modal phase speed corresponding to the horizontal wavenumbers $k_{r n}$ as $c_{n}=\omega / k_{r n}$, the normal mode solution in the fluid far from the source is found to be

$$
\phi_{1}(r, z)=\phi_{1}^{\prime}(r, z)=\frac{2 \pi}{H} \sqrt{\frac{2}{\pi r}} \sum_{n} \frac{1}{\sqrt{k_{r n}}} \mathrm{e}^{i\left(k_{r n} r-\pi / 4\right)} \Phi_{1}\left(k_{r n}\right) \sin \left(k_{z, 1} z_{s}\right) \sin \left(k_{z, 1} z\right), \quad 0 \leq z \leq H,
$$

where

$$
\Phi_{1}=-k_{r n} H \frac{\rho_{1}}{\rho_{2}} \frac{c_{n}^{4}}{c_{s}^{4}} \frac{k_{z, 2}}{k_{z, 1}}\left(\sqrt{c_{n}^{2} / c_{1}^{2}-1} M \cos \left(k_{z, 1} H\right)\right)^{-1}
$$

and

$$
\begin{aligned}
M= & \frac{\rho_{1}}{\rho_{2}} \frac{c_{n}^{4}}{c_{s}^{4}}\left\{\frac{\sin \left(k_{z, 1} H\right)}{\sqrt{c_{n}^{2} / c_{1}^{2}-1} \sqrt{1-c_{n}^{2} / c_{2}^{2}}}\left[1+\frac{1-c_{n}^{2} / c_{2}^{2}}{c_{n}^{2} / c_{1}^{2}-1}\right]-\left[\frac{k_{r n} H \sqrt{1-c_{n}^{2} / c_{2}^{2}}}{c_{n}^{2} / c_{1}^{2}-1} \sec \left(k_{z, 1} H\right)\right]\right\} \\
& -4\left\{\frac{\sqrt{1-c_{n}^{2} / c_{s}^{2}}}{\sqrt{1-c_{n}^{2} / c_{2}^{2}}}+\frac{\sqrt{1-c_{n}^{2} / c_{2}^{2}}}{\sqrt{1-c_{n}^{2} / c_{s}^{2}}}+2 \sqrt{1-c_{n}^{2} / c_{2}^{2}} \sqrt{1-c_{n}^{2} / c_{s}^{2}}-2\left(2-c_{n}^{2} / c_{s}^{2}\right)\right\} \\
& \times \cos \left(k_{z, 1} H\right) .
\end{aligned}
$$


Note that although the vertical wavenumber $k_{z, 1}$ has no subscript $n$, it is a function of $k_{r n}$ and so varies with $n$. The displacement potential solutions for $\phi_{2}(r, z)$ and $\psi(r, z)$ are not given here, as they are not needed for comparisons, but may be found in Refs. 4 or 7.

\section{The NRL experiment}

In April 2004, a series of scale-model tank experiments were performed, in part to test the validity of parabolic equation seismo-acoustic solutions. The experimental data collected was shown to have excellent agreement with an elastic parabolic equation solution for both range-dependent and range-independent environments. ${ }^{2}$ In the current work, only the range-independent case is considered. In the experiment, a large freshwater tank represented the ocean, and an elastic bottom was represented by a $122 \times 122 \times 10 \mathrm{~cm}$ PVC slab suspended in the water by cables. Material properties of the slab were estimated in a separate laboratory test. ${ }^{2}$ Source and receiver hydrophones were positioned over the slab using a robotic apparatus for accurate positioning. The source position was fixed and the receiver was moved in $2 \mathrm{~mm}$ increments away from the source at constant depth, creating a virtual aperture of 551 receiver elements. Experiments were performed using a broadband source whose frequency response was nominally flat between 100 and $300 \mathrm{kHz}$. Calculations with the normal mode solution are presented at a scale of 1000:1 in order to compare with the experimental data and to simulate an actual oceanic waveguide. For a more complete discussion of the experiment, the reader is referred to the paper by Collis et al. ${ }^{2}$

\section{Propagation calculations}

Geometric parameters used in normal mode solution calculations are from Ref. 2, specifically those determined through the use of a simulated annealing inversion scheme. Comparisons between the experimental data and the normal mode solutions are made using mid-depth and deep source positions and a deep receiver position. The depth of the PVC plate at the source was $14.47 \mathrm{~cm}$, while the depth of the slab edge near the end of the propagation track was $14.54 \mathrm{~cm}$. For normal mode calculations, the plate depth is approximated as flat, parallel to the water surface, at $14.50 \mathrm{~cm}$.

Solutions provided by normal mode calculations are highly sensitive to errors in horizontal wavenumber estimates. In order to accurately determine the complex horizontal wavenumbers the KRAKENC code is used. ${ }^{9}$ Using the parameters discussed previously, there are 19 wavenumbers corresponding to propagating modes for a $f=100 \mathrm{kHz}$ source; 39 propagating modes at $f=200 \mathrm{kHz}$; and 58 propagating modes at $f=300 \mathrm{kHz}$. Note that the horizontal wavenumbers are independent of source depth.

\section{Comparisons of measurements and calculations}

Data comparisons are made with the elastic Pekeris waveguide normal mode solution for the compressional field in the water, Eq. (12). Results are presented for select source frequencies of 100,200 , and $300 \mathrm{~Hz}$. For all calculations, the water sound speed is $1482 \mathrm{~m} / \mathrm{s}$ and the slab density, sound speeds, and attenuations are the measured values given in Table 1. Computations are made to the effective maximum range of

Table 1. Values for the PVC plate used in the NRL experiment estimated at $300 \mathrm{kHz}$.

\begin{tabular}{lr}
\hline \hline Parameter & Value \\
\hline Density $\left(\mathrm{g} / \mathrm{cm}^{3}\right)$ & 1.378 \\
Compressional speed (m/s) & 2290 \\
Compressional attenuation (dB/wavelength) & 0.76 \\
Shear speed $(\mathrm{m} / \mathrm{s})$ & 1050 \\
Shear attenuation $(\mathrm{dB} /$ wavelength) & 1.05 \\
\hline \hline
\end{tabular}


$120 \mathrm{~cm}$. All comparisons are made at the fixed receiver depth of $z_{r}=13.71 \mathrm{~cm}$ and two source depths: mid-depth, $z_{s}=6.91 \mathrm{~cm}$, and deep $z_{s}=13.68 \mathrm{~cm}$. Comparisons are shown for transmission loss (TL), calculated from the acoustic pressure in Eq. (1) as $\mathrm{TL}(r, z)=-20 \log _{10}\left(\operatorname{abs}\left(p(r, z) / p_{0}\right)\right)$, where $p_{0}$ is the pressure $1 \mathrm{~m}$ from the source.

For the mid-depth source case, Fig. 1 shows transmission loss versus range of the elastic Pekeris waveguide normal mode solution versus the experimental data. There is close agreement between the calculated elastic bottom solution and the experimental data. The solution follows even detailed variations in the transmission loss pattern. As the frequency increases, there are amplitude differences between the patterns closer to the source. Agreement between the elastic Pekeris waveguide solution and the experimental data moderately differs beyond approximately $106.8 \mathrm{~cm}$. This is due to the fact that the PVC plate ends, introducing range-dependent bathymetry, which is not accounted for by the normal mode solution.

For the deep source case, Fig. 2 shows transmission loss comparisons. Again, agreement is excellent between the normal mode solution and measured data. The transmission loss patterns are much more complicated due to source proximity to the bottom, yet the solution does a generally excellent job of reproducing the field. Amplitude differences are primarily observed near the source, and these increase with frequency. As the range from the source increases, these differences decrease. This suggests that the amplitude differences may be a result of the neglected mode continuum due to the proximity to the source, which is in actuality about $10 \mathrm{~cm}$ from the beginning of the propagation track.

To consider the effects of modal content on field calculations, Hankel transformations are taken of the complex pressure data to generate wavenumber spectra shown in Fig. 3. In Figs. 3(a) and 3(b), the elastic Pekeris solution closely matches the experimental modal content at both source positions for a $100 \mathrm{~Hz}$ source frequency. Note that below the compressional wavenumber for the bottom (approximately $0.27 \mathrm{~m}^{-1}$ ),
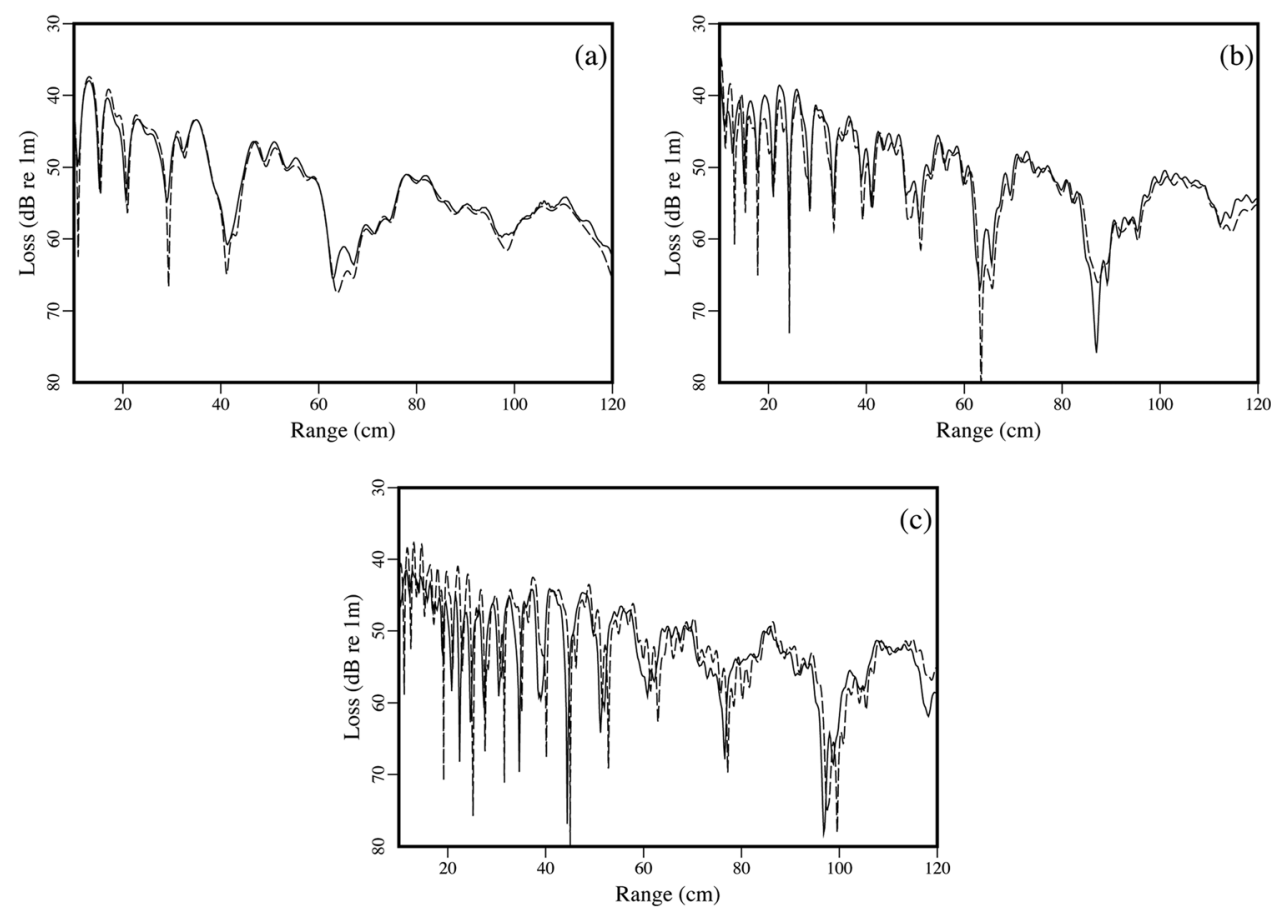

Fig. 1. Transmission loss versus range for the mid-depth source at $6.91 \mathrm{~cm}$ and near-bottom receiver at $13.71 \mathrm{~cm}$. Comparisons show data (solid curve) and calculations from the elastic Pekeris waveguide normal mode solution (dashed curve), for source frequencies: (a) $100 \mathrm{kHz}$, (b) $200 \mathrm{kHz}$, and (c) $300 \mathrm{kHz}$. 

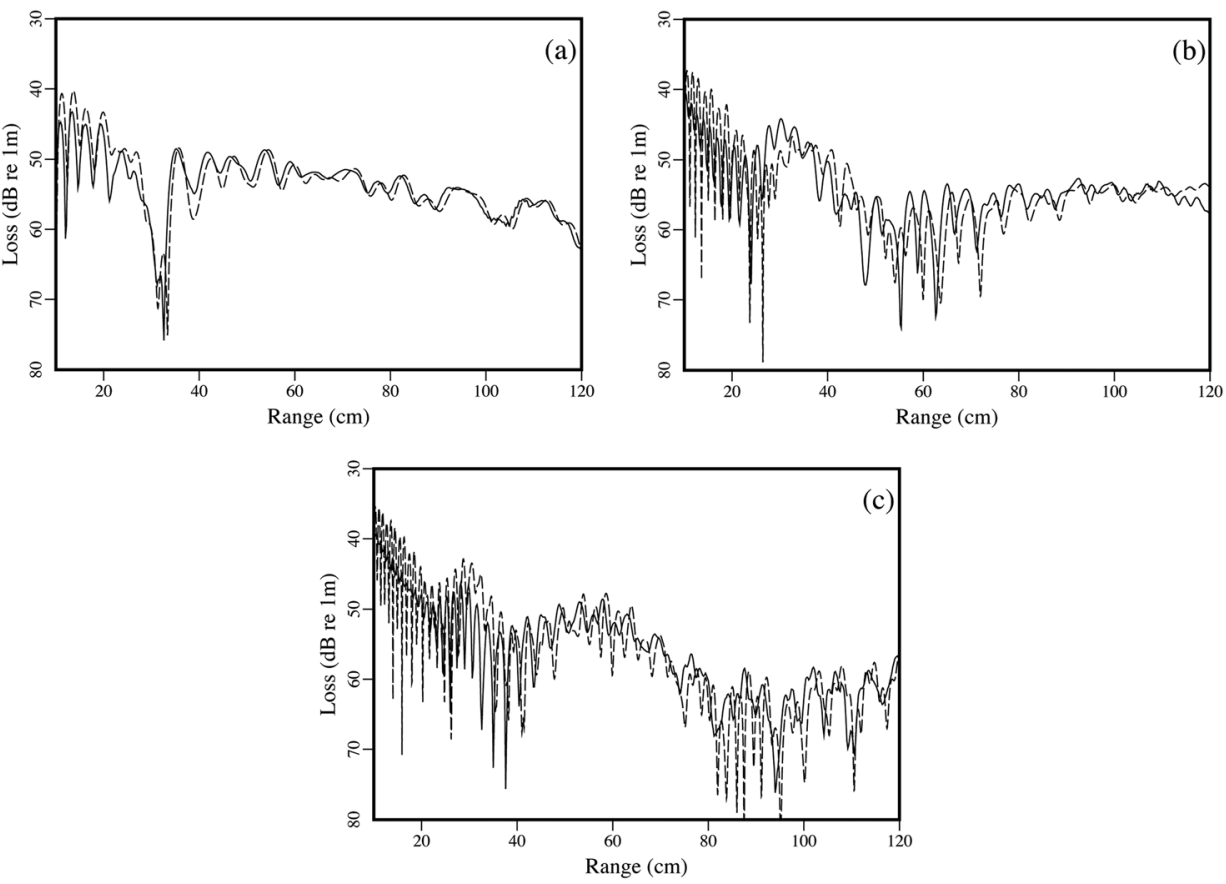

Fig. 2. Transmission loss versus range for the near-bottom source at $13.68 \mathrm{~cm}$ and near-bottom receiver at $13.71 \mathrm{~cm}$. Comparisons show data (solid curve) and calculations from the elastic Pekeris waveguide normal mode solution (dashed curve), for source frequencies: (a) $100 \mathrm{kHz}$, (b) $200 \mathrm{kHz}$, and (c) $300 \mathrm{kHz}$.
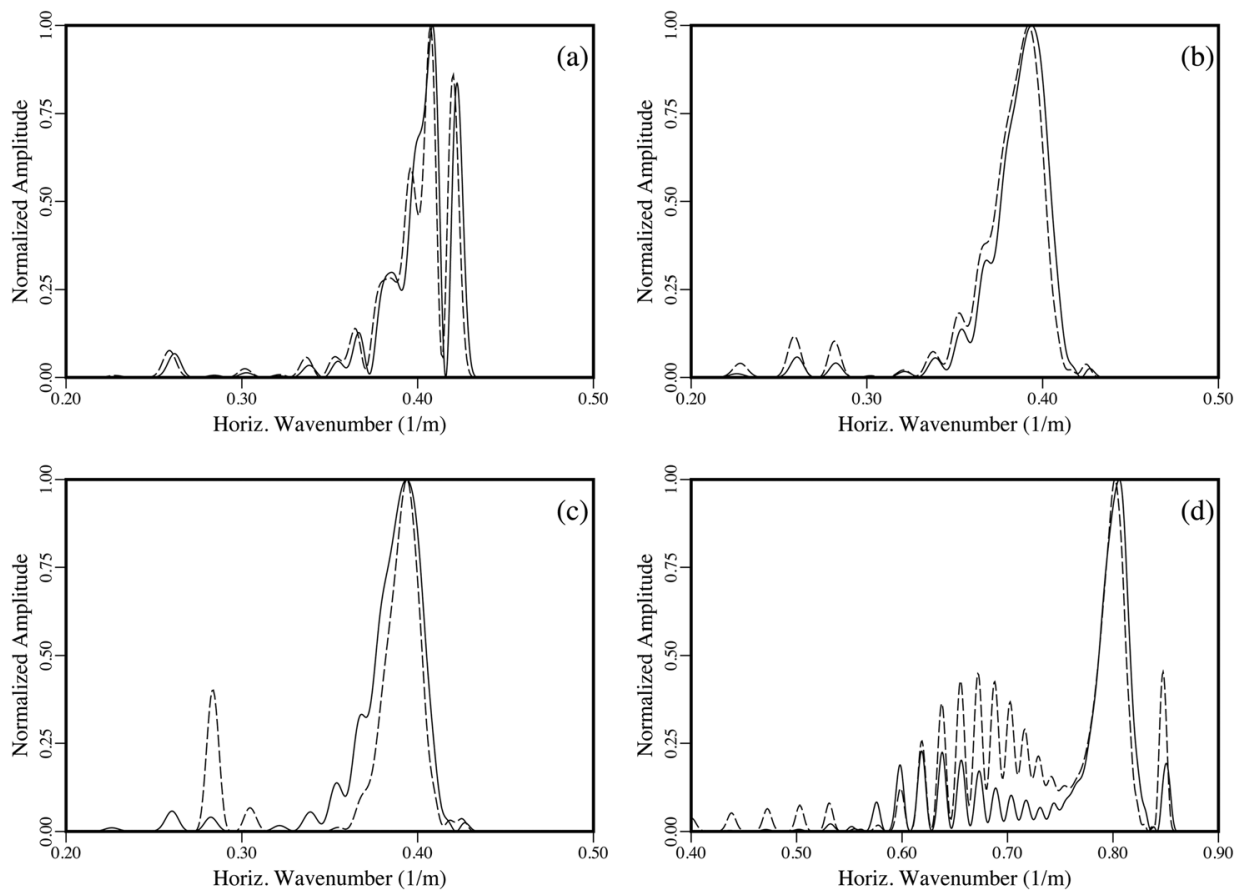

Fig. 3. Horizontal wavenumber spectra comparisons of experimental data versus fluid and elastic normal mode solutions. Comparisons show data (solid curve) and calculations from Pekeris waveguide normal mode solutions (dashed curve) for select configurations: (a) $100 \mathrm{~Hz}$, elastic solution, mid-depth source; (b) same as (a) except deep source; (c) same as (b) except fluid solution; (d) same as (b) except $200 \mathrm{~Hz}$. 
the data and elastic solutions indicate modes in the sediment. A spectrum from a fluid Pekeris waveguide solution ${ }^{3}$ is shown in Fig. 3(c) for the deep source case. The fluid solution does not capture all of the modal content when compared against the data; however, it does capture major aspects such as the dominant second mode and also the first compressional mode in the bottom. For a $200 \mathrm{~Hz}$ source [Fig. 3(d)], the wavenumber spectrum is more complex than for a $100 \mathrm{~Hz}$ source, yet the data and elastic solution capture compressional modes (below approximately $0.55 \mathrm{~m}^{-1}$ ) in the bottom. Note that for all cases, wavenumber spectra for the data and the simulations do not capture any explicit information about modes associated with the shear field in the bottom.

\section{Summary}

An adiabatic normal mode solution for the elastic Pekeris waveguide problem is benchmarked against experimental data, and solutions are shown to be in close agreement with the data. When effects due to elasticity are significant, the elastic Pekeris waveguide solution accurately represents range-independent shallow water environments. Note that these results are specific to solutions far from the source. Horizontal wavenumber spectra demonstrate that the elastic Pekeris waveguide solution captures the modal content of the pressure field well, although there are differences that may be used to explain discrepancies in field calculations. While modal content in the experimental data could be used to invert for compressional field properties, there is no evidence in this data that inversion could be performed for shear field properties.

\section{Acknowledgments}

This work was supported in part by the National Science Foundation Division of Graduate Education, NSF Grant No. DGE-0638719.

\section{References and links}

${ }^{1}$ E. K. Westwood, C. T. Tindle, and N. R. Chapman, "A normal mode model for acousto-elastic ocean environments," J. Acoust. Soc. Am. 100, 3631-3645 (1996).

${ }^{2}$ J. M. Collis, W. L. Siegmann, M. D. Collins, H. J. Simpson, and R. J. Soukup, "Comparison of simulations and data from a seismo-acoustic tank experiment,” J. Acoust. Soc. Am. 122, 1987-1993 (2007).

${ }^{3}$ C. L. Pekeris, "Theory of propagation of explosive sound in shallow water," in Propagation of Sound in the Ocean, Geol. Soc. Am., Mem. 27, 1-112 (1948).

${ }^{4} \mathrm{~F}$. Press and M. Ewing, "Propagation of explosive sound in a liquid layer over-lying a semi-infinite elastic solid," Geophysics. 15, 426-446 (1950).

${ }^{5} \mathrm{M}$. D. Collins, "A higher-order parabolic equation for wave propagation in an ocean overlying an elastic bottom,” J. Acoust. Soc. Am. 86, 1459-1464 (1989).

${ }^{6} \mathrm{~S}$. Ivansson and I. Karasalo, "A high-order adaptive integration method for wave propagation in rangeindependent fluid-solid media,” J. Acoust. Soc. Am. 92, 1569-1577 (1992).

${ }^{7}$ M. Ewing, W. Jardetzky, and F. Press, Elastic Waves in Layered Media (McGraw-Hill, New York, 1957), pp. 174-176.

${ }^{8}$ J. H. Mathews and R. W. Howell, Complex Analysis for Mathematics and Engineering, 4th ed. (Jones and Bartlett, Burlington, MA, 2001), pp. 309-311.

${ }^{9}$ M. B. Porter, "The KRAKEN Normal Mode Program," 1997, http://oalib.hlsresearch.com/Modes/ AcousticsToolbox/manual_html/kraken.html (last viewed October 5, 2011). 